\title{
Can DFT and ab initio methods describe all aspects of the potential energy surface of cycloreversion reactions?
}

\author{
Li-Juan Yu, ${ }^{a}$ Farzaneh Sarrami, ${ }^{a}$ Robert J. O'Reilly, ${ }^{\mathrm{b}}$ and Amir Karton ${ }^{\mathrm{a},}$ \\ ${ }^{a}$ School of Chemistry and Biochemistry, The University of Western Australia, Perth, WA 6009, \\ Australia. \\ ${ }^{b}$ Department of Chemistry, School of Science and Technology, Nazarbayev University, Astana, \\ Republic of Kazakhstan.
}

\begin{abstract}
A B S T R A C T
We introduce a representative benchmark database of 20 cycloreversion reaction energies obtained by means of the high-level W1 thermochemical protocol. We use these benchmark values to assess the performance of a variety of contemporary DFT, double-hybrid DFT (DHDFT), standard ab initio, and compound thermochemistry methods. We show that this set of reaction energies provides an extremely challenging test for nearly all of the considered DFT and DHDFT methods. For example, about $80 \%$ of the considered functionals result in root-meansquare deviations (RMSDs) above $10 \mathrm{~kJ} \mathrm{~mol}^{-1}$. The best DFT and DHDFT procedures are $\omega B 97 X$ and DSD-PBEP86-D3, with RMSDs of 4.7 and $7.9 \mathrm{~kJ} \mathrm{~mol}^{-1}$, respectively). Coupled with the fact that the barrier heights for these reactions also pose a significant challenge for many DFT methods, this work shows that only a handful of functionals can quantitatively describe all aspects of the potential surface of this important class of reactions. In addition, this work shows that London dispersion effects are particularly large for this class of reactions. For example, empirical dispersion corrections reduce the RMSDs for the DFT and DHDFT procedures by amounts ranging from 3.5 (PBE and B2K-PLYP) to 22.0 (BLYP) $\mathrm{kJ} \mathrm{mol}^{-1}$.
\end{abstract}

Keywords: cycloreversion $\bullet$ cycloelimination $\bullet$ density functional theory $\bullet$ D3 dispersion corrections $\bullet \mathrm{W} 1$ theory

How to cite this paper:

L.-J. Yu, F. Sarrami, R.J. O’Reilly, A. Karton. Mol. Phys. 114, 21 (2016).

Corresponding Author. Tel.: +61 86488 3139. Fax: +61 86488 7330, E-mail address:

amir.karton@uwa.edu.au (A. Karton). 


\section{Introduction}

Over the past two decades density functional theory (DFT) has become the most applied quantum chemical method due to its attractive accuracy-to-computational cost ratio. The approximations for the exchange-correlation functional can be classified according to their rung on Perdew's 'Jacob's Ladder':' (1) the local density approximation; (2) pure generalized gradient approximation (GGA) employing both the local density and the reduced density gradient; (3) the meta-GGAs which additionally employ the kinetic energy density; (4) the hybrid-GGAs and hybrid-meta-GGAs which in addition involve the occupied orbitals; and (5) the double-hybrid functionals which additionally employ the virtual orbitals. One weakness of the pure and hybridGGAs is that there is often a tradeoff between their performance for thermochemistry and thermochemical kinetics. ${ }^{2,3,4,5,6}$ For example, for hybrid-GGAs, the optimal percentage of Hartree-Fock exchange for thermochemical properties tends to be around the $\sim 20 \%$ mark, whilst for thermochemical kinetics it tends to be at least twice as much. ${ }^{3,4,5,7,8,9}$ Hybrid-meta GGA and double-hybrid procedures can overcome this deficiency to a considerable extent. ${ }^{5,9,10,11,12}$ However, it is important to identify reactions for which these more sophisticated fuctionals fail to offer a quantitative description of both the reaction energies and barrier heights. Identifying these problematic reactions is important since (i) DFT functionals should be applied with caution for modeling these reactions, and (ii) it may assist in the development of next-generation functionals with improved performance.

Cycloreversion of heteroatom rings, in which a cyclic structure is fragmented into simple unsaturated building blocks, is an important class of organic reactions which are widely used in synthetic and medicinal chemistry. ${ }^{13,14,15,16}$ We have recently evaluated the performance of a range of contemporary DFT methods for the reaction barrier heights of 20 cycloreversion reactions of 5-membered heterocyclic rings (known as the CRBH20 dataset). ${ }^{17}$ These reaction barrier heights provide a challenging test for DFT and double-hybrid DFT (DHDFT) methods. For example, of the 65 tested DFT and DHDFT functionals, only two DHDFT functionals (B2KPLYP and DSD-PBEP86) result in root-mean-square deviations (RMSDs) below the 'chemical accuracy' threshold ('chemical accuracy' is arbitrarily defined here as RMSDs $\leq 4.2 \mathrm{~kJ} \mathrm{~mol}^{-1}$ from accurate reference data).

The present study aims to answer an important question: can DFT methods quantitatively describe all aspects of the potential energy surfaces of cycloreversion reactions? (i.e., exhibit reasonably good performance for both reaction energies and barrier heights). To this end, we use a more lenient cutoff of twice the threshold of chemical accuracy (i.e., RMSD $\leq 8.4 \mathrm{~kJ} \mathrm{~mol}^{-1}$ ) to indicate that a functional is performing reasonably well for these challenging reactions. We show 
that only a handful of DFT and DHDFT functionals exhibit reasonable performance for the calculation of both the energies and barrier heights of cycloreversion reactions. For example, only 6 of the 65 considered DFT functionals exhibit RMSDs $\leq 8.4 \mathrm{~kJ} \mathrm{~mol}^{-1}$ for both the reaction energies and barrier heights (namely, 3 hybrid GGA, 2 hybrid-meta GGA, and 1 double-hybrid functional). This result is significant because it demonstrates that even sophisticated functionals from rungs four and five of Jacob's Ladder cannot quantitatively describe all aspects of the potential surface of this important class of reactions.

\section{Computational Methods}

We evaluate the performance of a large number of DFT and ab initio procedures for a set of 20 cycloreversion reaction energies (to be known as the CR20 dataset). In order to obtain reliable reference reaction energies, calculations have been carried out using the high-level, ab initio W1w theory ${ }^{18,19}$ or its explicitly correlated version (W1-F12 theory) ${ }^{20}$ with the Molpro 2012.1 program suite. ${ }^{21,22}$ These theories represent layered extrapolations to the all-electron $\operatorname{CCSD}(\mathrm{T}) / \mathrm{CBS}$ limit, ${ }^{23,24}$ and can achieve 'sub-chemical accuracy' for atomization reactions. ${ }^{25}$ For example, W1w and W1-F12 theories are associated with RMSDs of 2.6 and $3.1 \mathrm{~kJ} \mathrm{~mol}^{-1}$ for a set of 140 very accurate atomization energies obtained at the full configuration interaction (FCI) infinite basis-set limit. ${ }^{18,20,25}$ Nevertheless, we point out that for systems containing only first-row elements (and H) W1-F12 shows better performance. Specifically, for the 97 first-row atomization energies in the W4-11 dataset, ${ }^{25} \mathrm{~W} 1-\mathrm{F} 12$ attains an RMSD of $1.9 \mathrm{~kJ} \mathrm{~mol}^{-1}$ relative to reference atomization energies at the FCI infinite basis-set limit. ${ }^{20}$ Therefore in the present work we obtain reaction energies with W1-F12 theory for systems containing only first-row elements and with W1w theory for systems containing second-row elements. For the sake of making the article self-contained, we will briefly outline the various steps in W1w theory (for further details see Refs. 18 and 19). The Hartree-Fock (HF) component is extrapolated from the A'VTZ and $\mathrm{A}^{\prime} \mathrm{VQZ}$ basis sets, using the $E(L)=E_{\infty}+A / L^{\alpha}$ two-point extrapolation formula with $\alpha=5$. The notation $\mathrm{A}^{\prime} \mathrm{V} n \mathrm{Z}$ indicates the combination of the standard correlation-consistent cc-p VnZ basis sets on $\mathrm{H}^{26}{ }^{26}$ the aug-cc-pVnZ basis sets on first-row atoms, ${ }^{27}$ and the aug-cc-pV( $\left.n+\mathrm{d}\right) \mathrm{Z}$ basis sets on second-row atoms. ${ }^{28}$ The valence CCSD correlation energy is extrapolated from the same basis sets with $\alpha=3.22$. The quasiperturbative triples, $(\mathrm{T})$, correction is extrapolated from the $\mathrm{A}^{\prime} \mathrm{VDZ}$ and $\mathrm{A}^{\prime} \mathrm{VTZ}$ basis sets with $\alpha=3.22$. The $\operatorname{CCSD}(\mathrm{T})$ inner-shell contribution is calculated with the MTsmall basis set, which is a completely decontracted cc-pVTZ basis set with tight $2 \mathrm{~d} 1 \mathrm{f}$ functions added. ${ }^{18} \mathrm{~W} 1-\mathrm{F} 12$ theory is an explicitly correlated version of W1w, which combines 
explicitly correlated F12 techniques with basis-set extrapolations in order to approximate the $\operatorname{CCSD}(\mathrm{T}) / \mathrm{CBS}$ energy. The computational protocol of W1-F12 theory has been specified and rationalized in detail in Ref. 20 (for a concise summary of the various steps in W1-F12 theory see Ref. 29).

The geometries of all structures have been obtained at the B3LYP/A'VTZ level of theory. ${ }^{30,31,32}$ Harmonic vibrational analyses have been performed to confirm each stationary point as an equilibrium structure (i.e., consisting of all real frequencies). All geometry optimizations and frequency calculations were performed using the Gaussian 09 program suite. ${ }^{33}$

The DFT exchange-correlation functionals considered in the present study (ordered by their rung on Jacob's Ladder) ${ }^{1}$ are the pure generalized gradient approximation (GGA) functionals: BLYP,${ }^{30,34}$ B97-D,${ }^{35}$ HCTH407, ${ }^{36}$ PBE,${ }^{37}$ BP86, ${ }^{34,38}$ BPW91, ${ }^{34,39}$ SOGGA11, ${ }^{40}$ N12; ${ }^{41}$ the metaGGAs (MGGAs): M06-L, ${ }^{42}$ TPSS, ${ }^{43} \tau$-HCTH, ${ }^{44} \mathrm{VSXC}^{45}{ }^{45 B} \mathrm{~B} 9,{ }^{46} \mathrm{M} 11-\mathrm{L},{ }^{47} \mathrm{MN} 12-\mathrm{L} ;{ }^{48}$ the hybrid-GGAs (HGGAs): BH\&HLYP, ${ }^{49}$ B3LYP, ${ }^{30,31,32}$ B3P86, ${ }^{31,38}$ B3PW91, ${ }^{31,39}$ PBE0, ${ }^{50}$ B97$1,{ }^{51} \mathrm{~B} 98,{ }^{52}$ X3LYP, ${ }^{53}$ SOGGA11-X; ${ }^{54}$ the hybrid-meta-GGAs (HMGGAs): M05, ${ }^{55} \mathrm{M} 05-2 \mathrm{X},{ }^{56}$ M06, ${ }^{9}$ M06-2X, ${ }^{9}$ M06-HF, ${ }^{9}$ BMK, ${ }^{3}$ B1B95, ${ }^{34,46}$ TPSSh, $^{57} \tau$-HCTHh, ${ }^{44}$ PW6B95, ${ }^{58}$ and the DHDFT procedures: ${ }^{11}$ B2-PLYP,${ }^{59}$ B2GP-PLYP,${ }^{10}$ B2K-PLYP,${ }^{60}$ B2T-PLYP,${ }^{60}$ DSD-BLYP,${ }^{61}$ DSD-PBEP86, ${ }^{11,62}$ and PWPB95. ${ }^{63}$ In addition to the global HGGAs and HMGGAs, we also consider the following range-separated (RS) functionals: CAM-B3LYP, ${ }^{64} \mathrm{LC}-\omega \mathrm{PBE},{ }^{65} \omega \mathrm{B} 97,{ }^{66}$ $\omega \mathrm{B} 97 \mathrm{X},{ }^{66} \omega \mathrm{B} 97 \mathrm{X}-\mathrm{D},{ }^{67}$ and M11 ${ }^{68}$ Empirical D3 dispersion corrections ${ }^{69,70,71}$ are included in some cases using the Becke-Johnson ${ }^{72}$ damping potential as recommended in Ref. 69 (denoted by the suffix -D3). We note that the suffix -D in B97-D and 6 B97X-D indicates the original dispersion correction rather than the D3 correction. The standard DFT calculations were carried out in conjunction with the $\mathrm{A}^{\prime} \mathrm{VTZ}$ basis set, while the DHDFT calculations, which exhibit slower basis set convergence, were carried out in conjunction with the A'VQZ basis set. All the DFT and DHDFT were performed using the Gaussian $09^{33}$ and $\mathrm{ORCA}^{73,74}$ program suites.

In addition, the performance of composite thermochemical procedures and standard ab initio methods are also assessed. We consider the following composite procedures: G4, ${ }^{75}$ G4(MP2), ${ }^{76}$ G4(MP2)-6X, ${ }^{77} \mathrm{CBS}-\mathrm{QB} 3,{ }^{78}$ and CBS-APNO,${ }^{79}$ and the following ab initio methods: MP2, SCS-MP2, ${ }^{80} \mathrm{MP} 2.5,{ }^{81} \mathrm{MP} 3, \mathrm{MP} 3.5,{ }^{82} \mathrm{SCS}-\mathrm{MP} 3,{ }^{83} \mathrm{MP} 4, \mathrm{MP} 4(\mathrm{SDQ}), \mathrm{MP}_{\mathrm{av}}{ }^{17} \mathrm{CCSD}$, SCS-CCSD, ${ }^{84} \mathrm{SCS}(\mathrm{MI}) \mathrm{CCSD},{ }^{85}$ and $\operatorname{CCSD}(\mathrm{T})$. The performance of the MP2- and MP3-based procedures is evaluated in conjunction with the $\mathrm{A}^{\prime} \mathrm{VQZ}$ basis set whereas the performance of the computationally more expensive MP4-based and coupled cluster procedures is evaluated in conjunction with the $\mathrm{A}^{\prime} \mathrm{VTZ}$ basis set. 


\section{Results and Discussion}

3.1. Overview of the benchmark reaction energies in the CR20 database. A schematic representation of the 20 cycloreversion reactions in the CR20 database is given in Figure 1, whilst the heteroatom $(\mathrm{X})$ and substituents $\left(\mathrm{R}_{1}-\mathrm{R}_{3}\right)$ are listed in Table 1. All the cycloreversion reactions involve cleavage of a heterocyclic ring into two unsaturated fragments. In addition, these reactions involve a migration of the $\mathrm{R}_{1}$ substituent across the $\mathrm{C}=\mathrm{N}$ bond (Fig. 1). There are two types of reactions in the CR20 dataset (Table 1):

i) Reactions 1-10 $(\mathrm{X}=\mathrm{O})$ are cycloreversions of dioxazoles forming carbonyls and isocyanates

ii) Reactions 11-20 $(\mathrm{X}=\mathrm{S})$ are cycloreversions of oxathiazoles forming carbonyls and isothiocyanates

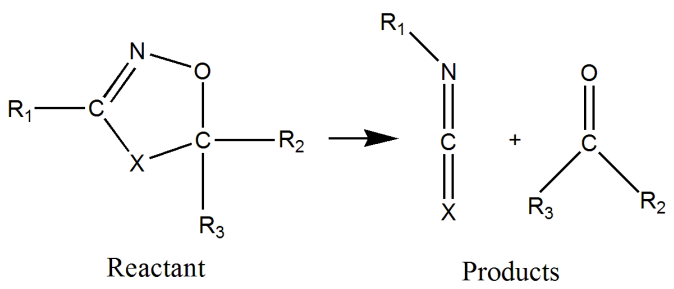

Figure 1. Schematic representation of the reactions in the CR20 database. The $X$ center and the $R_{1}, R_{2}$, and $R_{3}$ functional groups are listed in Table 1 . 
Table 1. Cycloreversion reactions in the CR20 database (see also Fig. 1).

\begin{tabular}{|c|c|c|c|c|}
\hline Reaction & $\mathbf{X}$ & $\mathbf{R}_{1}$ & $\mathbf{R}_{2}$ & $\mathbf{R}_{\mathbf{3}}$ \\
\hline 1 & $\mathrm{O}$ & $\mathrm{H}$ & $\mathrm{H}$ & $\mathrm{H}$ \\
\hline 2 & $\mathrm{O}$ & $\mathrm{Me}$ & $\mathrm{H}$ & $\mathrm{H}$ \\
\hline 3 & $\mathrm{O}$ & $\mathrm{Et}$ & $\mathrm{H}$ & $\mathrm{H}$ \\
\hline 4 & $\mathrm{O}$ & $\mathrm{CH}_{2} \mathrm{~F}$ & $\mathrm{H}$ & $\mathrm{H}$ \\
\hline 5 & $\mathrm{O}$ & $\mathrm{Me}$ & $\mathrm{Me}$ & $\mathrm{H}$ \\
\hline 6 & $\mathrm{O}$ & $\mathrm{Me}$ & $\mathrm{OH}$ & $\mathrm{H}$ \\
\hline 7 & $\mathrm{O}$ & $\mathrm{Me}$ & $\mathrm{NH}_{2}$ & $\mathrm{H}$ \\
\hline 8 & $\mathrm{O}$ & $\mathrm{Me}$ & $\mathrm{F}$ & $\mathrm{H}$ \\
\hline 9 & $\mathrm{O}$ & $\mathrm{Me}$ & $\mathrm{Me}$ & $\mathrm{Me}$ \\
\hline 10 & $\mathrm{O}$ & $\mathrm{Me}$ & & \\
\hline 11 & S & $\mathrm{H}$ & $\mathrm{H}$ & $\mathrm{H}$ \\
\hline 12 & $\mathrm{~S}$ & $\mathrm{Me}$ & $\mathrm{H}$ & $\mathrm{H}$ \\
\hline 13 & $\mathrm{~S}$ & Et & $\mathrm{H}$ & $\mathrm{H}$ \\
\hline 14 & $\mathrm{~S}$ & $\mathrm{CH}_{2} \mathrm{~F}$ & $\mathrm{H}$ & $\mathrm{H}$ \\
\hline 15 & S & $\mathrm{Me}$ & $\mathrm{Me}$ & $\mathrm{H}$ \\
\hline 16 & S & $\mathrm{Me}$ & $\mathrm{OH}$ & $\mathrm{H}$ \\
\hline 17 & S & $\mathrm{Me}$ & $\mathrm{NH}_{2}$ & $\mathrm{H}$ \\
\hline 18 & $\mathrm{~S}$ & $\mathrm{Me}$ & $\mathrm{F}$ & $\mathrm{H}$ \\
\hline 19 & S & $\mathrm{Me}$ & $\mathrm{Me}$ & $\mathrm{Me}$ \\
\hline 20 & S & $\mathrm{Me}$ & & \\
\hline
\end{tabular}

${ }^{a}$ The $\mathrm{CR}_{2} \mathrm{R}_{3}$ moiety is replaced with a $\mathrm{C}=\mathrm{O}$ group (see Fig. 1 ).

The species in the CR20 database cover a broad spectrum of substituents and bonding situations. We consider two parent structures in which $\mathrm{R}_{1}=\mathrm{R}_{2}=\mathrm{R}_{3}=\mathrm{H}$ (and Me). In addition, the $R_{1}, R_{2}$, and $R_{3}$ substituents have been varied such that: (i) $R_{2}=H, M e, N_{2}, O H$, and $F$, whilst maintaining a constant migrating group (i.e., $\mathrm{R}_{1}=\mathrm{Me}$ ), and (ii) the migrating group is varied $\left(\mathrm{R}_{1}=\mathrm{H}\right.$, Me, Et, and $\left.\mathrm{CH}_{2} \mathrm{~F}\right)$, whilst maintaining $\mathrm{R}_{2}$ and $\mathrm{R}_{3}$ constant (i.e., $\mathrm{R}_{2}=\mathrm{R}_{3}=H$ ). In addition, we consider the possibility of replacing the $\mathrm{CR}_{2} \mathrm{R}_{3}$ moiety with a carbonyl group.

Benchmark reference data have been obtained by means of the high-level W1-F12 and W1w procedures. ${ }^{18,19,20}$ Since these procedures represent layered extrapolations to the CCSD(T) basis-set-limit energy, it is of interest to estimate whether the contributions from post-CCSD(T) excitations are likely to be significant. The percentage of the total atomization energy accounted for by parenthetical connected triple excitations, \% $\mathrm{TAE}_{e}[(\mathrm{~T})]$, has been shown to be a reliable energy-based diagnostic for the importance of post-CCSD(T) contributions. It has been suggested that $\% \mathrm{TAE}_{e}[(\mathrm{~T})]<2$ indicates systems that are dominated by dynamical correlation, while $2<$ $\% \operatorname{TAE}_{e}[(\mathrm{~T})]<5$ indicates systems that include mild nondynamical correlation. ${ }^{19,25,86}$ Table S1 (Supplementary data) gathers the $\% \mathrm{TAE}_{e}[(\mathrm{~T})]$ values for the reactants and products involved in 
the CR20 dataset. The \% $\operatorname{TAE}_{e}[(\mathrm{~T})]$ values for these species lie in the range 1.6-3.9 (where in $87 \%$ of cases the $\left.\% \operatorname{TAE}_{e}[(\mathrm{~T})] \leq 3.0\right)$. These values suggest that the $\operatorname{CCSD}(\mathrm{T})$ method is adequate for the description of the reaction energies in the CR20 database and that our bottom-of-the-well CCSD(T)/CBS benchmark values should be well below $1 \mathrm{kcal} \mathrm{mol}^{-1}$ from the full configuration interaction (FCI) basis-set limit. ${ }^{20,25,87}$

The component breakdown of the $\mathrm{W} 1-\mathrm{F} 12$ and $\mathrm{W} 1 \mathrm{w}$ reaction energies are gathered in Table 2. All the reactions in the CR20 database are exothermic with reaction energies ranging from -32.0 (12) to $-149.4(\mathbf{1 0}) \mathrm{kJ} \mathrm{mol}^{-1}$. The reactions of the dioxazole systems are systematically more exothermic than those of the oxathiazoles (by amounts ranging from 8.7$56.9 \mathrm{~kJ} \mathrm{~mol}^{-1}$ ). For both the dioxazoles and oxathiazoles the most exothermic reaction is obtained when $\mathrm{R}_{1}$ is a methyl group and $\mathrm{CR}_{2} \mathrm{R}_{3}$ is a $\mathrm{C}=\mathrm{O}$ group, namely $\Delta E_{e}=-149.4$ (dioxazole, 10) and -133.1 (oxathiazole, 20) $\mathrm{kJ} \mathrm{mol}^{-1}$. Similarly, for both ring types the least exothermic reaction is obtained for $\mathrm{R}_{1}=\mathrm{Me}$ and $\mathrm{R}_{2}=\mathrm{R}_{3}=\mathrm{H}$, namely $\Delta E_{e}=-67.6$ (dioxazole, 2) and -32.0 (oxathiazole, 12) $\mathrm{kJ} \mathrm{mol}^{-1}$.

Table 2. Component breakdown of the benchmark W1-F12 and $\mathrm{W} 1 \mathrm{w}$ reaction energies for the reactions in the CR20 database (in $\mathrm{kJ} \mathrm{mol}^{-1}$, see Table 1 and Fig. 1 for the reaction definitions). ${ }^{a}$

\begin{tabular}{cccccc}
\hline Reaction & $\Delta \mathrm{SCF}$ & $\Delta \mathrm{CCSD}$ & $\Delta(\mathrm{T})$ & $\Delta \mathrm{CV}^{b}$ & $\Delta \mathrm{E}_{e}{ }^{c}$ \\
\hline $\mathbf{1}$ & -182.6 & 49.6 & 3.7 & -1.9 & -131.2 \\
$\mathbf{2}$ & -122.6 & 52.9 & 3.8 & -1.7 & -67.6 \\
$\mathbf{3}$ & -133.1 & 54.5 & 4.1 & -1.8 & -76.3 \\
$\mathbf{4}$ & -158.2 & 55.6 & 4.3 & -1.6 & -99.9 \\
$\mathbf{5}$ & -135.2 & 58.1 & 4.5 & -1.8 & -74.4 \\
$\mathbf{6}$ & -177.3 & 55.7 & 3.5 & -2.1 & -120.3 \\
$\mathbf{7}$ & -189.5 & 59.3 & 3.9 & -2.5 & -128.8 \\
$\mathbf{8}$ & -141.8 & 50.6 & 2.5 & -2.0 & -90.7 \\
$\mathbf{9}$ & -147.8 & 64.2 & 5.8 & -1.9 & -79.7 \\
$\mathbf{1 0}$ & -206.4 & 55.5 & 3.7 & -2.1 & -149.4 \\
$\mathbf{1 1}$ & -131.5 & 54.7 & 4.0 & -1.5 & -74.3 \\
$\mathbf{1 2}$ & -99.9 & 63.2 & 6.2 & -1.5 & -32.0 \\
$\mathbf{1 3}$ & -111.2 & 65.7 & 6.8 & -1.5 & -40.2 \\
$\mathbf{1 4}$ & -117.0 & 61.7 & 4.9 & -1.3 & -51.6 \\
$\mathbf{1 5}$ & -124.0 & 71.0 & 8.0 & -1.7 & -46.8 \\
$\mathbf{1 6}$ & -181.2 & 69.2 & 7.2 & -1.9 & -106.7 \\
$\mathbf{1 7}$ & -182.2 & 72.6 & 7.4 & -2.4 & -104.5 \\
$\mathbf{1 8}$ & -149.1 & 63.2 & 5.8 & -1.9 & -82.0 \\
$\mathbf{1 9}$ & -144.0 & 78.4 & 9.7 & -1.9 & -57.7 \\
$\mathbf{2 0}$ & -210.1 & 70.3 & 8.7 & -2.1 & -133.1 \\
\hline
\end{tabular}

${ }^{a}$ The reference values for reactions 1-10 are from W1-F12 theory, and for reactions $\mathbf{1 1 - 2 0}$ from $\mathrm{W} 1 \mathrm{w}$ theory. ${ }^{b} \mathrm{Core}-$ valence correction. ${ }^{c}$ All-electron, vibrationless $\operatorname{CCSD}(\mathrm{T})$ basis-set-limit reaction energies. 
Inspection of Table 2, reveals that the HF/CBS level of theory systematically underestimates the reaction energies by amounts ranging from 51.1 (8) to $86.3(\mathbf{1 9}) \mathrm{kJ} \mathrm{mol}^{-1}$ relative to the $\operatorname{CCSD}(\mathrm{T}) / \mathrm{CBS}$ reference values. The valence $\mathrm{CCSD}$ correlation contribution to the reaction energies increases the reaction energies by amounts ranging between 49.6 (1) and 78.4 (19) $\mathrm{kJ} \mathrm{mol}^{-1}$. The quasiperturbative triples, $(\mathrm{T})$, contribution further increases the reaction energies by chemically significant amounts ranging from $2.5(8)$ and $9.7(\mathbf{1 9}) \mathrm{kJ} \mathrm{mol}^{-1}$. Finally, we note that the core-valence correlation contribution systematically reduces the reaction energies by relatively modest amounts ranging from $1.3(\mathbf{1 4})$ to $2.5(7) \mathrm{kJ} \mathrm{mol}^{-1}$.

\subsection{Performance of standard and composite ab initio procedures for the reaction energies}

in the CR20 database. Table 3 gives an overview of the performance of the composite G3, G3(MP2), G4, G4(MP2), G4(MP2)-6X, CBS-QB3, and CBS-APNO procedures, as well as several ab initio methods (e.g., MP2, MP2.5, MP3, MP3.5, MP4, SCS-MP2, SCS-MP3, CCSD and $\operatorname{CCSD}(\mathrm{T})$ ). Inspection of Table 3 reveals that most of the composite procedures do not have difficulties with the reactions in the CR20 dataset. The CBS-type procedures show excellent performance with RMSDs of 2.9 (CBS-QB3) and 3.4 (CBS-APNO) $\mathrm{kJ} \mathrm{mol}^{-1}$. Three of the $\mathrm{G} n$ type procedures result in RMSDs below the 'chemical accuracy' threshold. Namely, G3 and G4 with an RMSD of $4.1 \mathrm{~kJ} \mathrm{~mol}^{-1}$ and G4(MP2)-6X with a slightly lower RMSD of $3.8 \mathrm{~kJ} \mathrm{~mol}^{-1}$. The rest of the $\mathrm{G} n$ procedures result in RMSDs ranging between 4.7 (G3(MP2)) and 7.4 (G3(MP2)B3) $\mathrm{kJ} \mathrm{mol}^{-1}$. Finally, we note that, apart from CBS-APNO, all the composite procedures tend to systematically underestimate the reaction energies. 
Table 3. Statistical analysis for the performance of composite and standard ab initio methods for the calculation of the reaction energies in the CR20 database (in $\mathrm{kJ} \mathrm{mol}^{-1}$ ).

\begin{tabular}{|c|c|c|c|c|c|}
\hline Basis set & Methods & $\mathrm{RMSD}^{a}$ & $\mathrm{MAD}^{a}$ & $\mathrm{MSD}^{a}$ & $\mathrm{LD}^{a, b}$ \\
\hline & G3 & 4.1 & 3.5 & -2.9 & $7.9(\mathbf{1 3})$ \\
\hline & G3(MP2) & 5.0 & 4.4 & -4.1 & $9.2(13)$ \\
\hline & G3B3 & 6.7 & 6.2 & -6.1 & $10.6(\mathbf{1 2})$ \\
\hline & G3(MP2)B3 & 7.4 & 6.9 & -6.9 & $11.0(\mathbf{1 2})$ \\
\hline & G4 & 4.1 & 3.8 & -3.6 & $6.5(12)$ \\
\hline & G4(MP2) & 4.7 & 4.3 & -4.3 & 7.4 (14) \\
\hline & G4(MP2)-6X & 3.8 & 3.4 & -3.1 & $6.9(14)$ \\
\hline & CBS-QB3 & 2.9 & 2.3 & -1.5 & $6.0(12)$ \\
\hline & $\mathrm{CBS}-\mathrm{APNO}^{c}$ & 3.4 & 1.6 & 3.2 & $5.8(9)$ \\
\hline \multirow[t]{6}{*}{$\mathrm{A}^{\prime} \mathrm{VQZ}$} & $\mathrm{HF}$ & 65.1 & 64.4 & -64.4 & 85.8 (19) \\
\hline & MP2 & 14.8 & 14.0 & -14.0 & $25.8(\mathbf{1 0})$ \\
\hline & SCS-MP2 & 23.4 & 23.1 & -23.1 & $32.3(\mathbf{1 0})$ \\
\hline & MP2.5 & 3.6 & 3.2 & -3.2 & $8.5(\mathbf{2 0})$ \\
\hline & MP3 & 8.6 & 7.6 & 7.6 & $13.8(8)$ \\
\hline & SCS-MP3 & 20.7 & 20.5 & -20.5 & $27.6(\mathbf{1 0})$ \\
\hline \multirow[t]{9}{*}{$\mathrm{A}^{\prime} \mathrm{VTZ}$} & MP3.5 & 2.4 & 2.0 & 1.6 & $4.4(9)$ \\
\hline & MP4(SDQ) & 4.8 & 4.6 & -4.6 & $6.8(\mathbf{2 0})$ \\
\hline & MP4(SDTQ) & 6.9 & 6.5 & -6.5 & $12.4(\mathbf{1 0})$ \\
\hline & $\mathrm{MP} 4_{\mathrm{av}}{ }^{d}$ & 5.7 & 5.6 & -5.6 & $8.3(\mathbf{1 0})$ \\
\hline & CCSD & 2.4 & 2.0 & -0.6 & $4.2(\mathbf{2 0})$ \\
\hline & SCS-CCSD & 12.4 & 12.2 & 12.2 & $16.0(7)$ \\
\hline & SCS(MI)CCSD & 10.9 & 10.7 & 10.7 & $13.6(7)$ \\
\hline & $\operatorname{CCSD}(\mathrm{T})$ & 4.7 & 4.4 & 4.4 & $6.8(9)$ \\
\hline & $\operatorname{CCSD}(\mathrm{T}) / \mathrm{CBS}(\mathrm{MP} 2)^{e}$ & 4.5 & 4.1 & 4.1 & $7.2(17)$ \\
\hline
\end{tabular}

${ }^{a} \mathrm{RMSD}=$ root-mean-square deviation, MAD = mean-absolute deviation, MSD = mean-signed deviation, LD = largest deviation (in absolute value). ${ }^{b}$ The reaction numbers are given in parenthesis (see also Table 1). ${ }^{c}$ Error statistics over the dioxazole systems only. ${ }^{d} \mathrm{MP} 4_{\mathrm{av}}$ is defined as the average of MP4(SDQ) and MP4(SDTQ), see Ref. 17 for further details. ${ }^{e} \mathrm{CCSD}(\mathrm{T}) / \mathrm{CBS}(\mathrm{MP} 2) \approx \mathrm{CCSD}(\mathrm{T}) / \mathrm{A}^{\prime} \mathrm{VDZ}+\mathrm{MP} 2 / \mathrm{A}^{\prime} \mathrm{V}\{\mathrm{T}, \mathrm{Q}\} Z-\mathrm{MP} 2 / \mathrm{A}^{\prime} \mathrm{VDZ}$.

We now turn our attention to the performance of the standard wavefunction methods. Table 3 gives these results in conjunction with the A'VQZ for the computationally more economical MP2- and MP3-based methods, whilst the performance of the MP4-based and coupled cluster methods is evaluated in conjunction with the A'VTZ basis set. Table S2 of the Supplementary data gives results for all the standard ab initio methods in conjunction with the $\mathrm{A}^{\prime} \mathrm{V} n \mathrm{Z}$ basis sets $(n=\mathrm{D}$ and $\mathrm{T})$. We start by noting that for the methods for which we have both $\mathrm{A}^{\prime} \mathrm{VTZ}$ and $\mathrm{A}^{\prime} \mathrm{VQZ}$ results (HF, MP2, SCS-MP2, MP2.5, MP3, and SCS-MP3) the difference in the overall RMSDs between the $\mathrm{A}^{\prime} \mathrm{VTZ}$ and $\mathrm{A}^{\prime} \mathrm{VQZ}$ basis sets are smaller than $2.1 \mathrm{~kJ} \mathrm{~mol}^{-1}$. However, in nearly all cases the $\mathrm{A}^{\prime} \mathrm{VTZ}$ basis set performs better than the $\mathrm{A}^{\prime} \mathrm{VQZ}$ basis set (relative to the W1-F12 and W1w reference values). For the most part, this results from error compensation between basis set incompleteness and the neglect of higher-level correlation effects. 
Inspection of Table 3 reveals that the CR20 database is a challenging target for nearly all of the standard ab initio methods. Only three of the 15 examined procedures result in RMSDs below the 'chemical accuracy' threshold (MP2.5, MP3.5, and CCSD). As mentioned in Section 3.1, Hartree-Fock theory systematically and severely underestimates the W1-F12 and W1w reaction energies. For the HF/A'VQZ level of theory we obtain an RMSD of $65.1 \mathrm{~kJ} \mathrm{~mol}^{-1}$ and an $\mathrm{MSD}=-1 \times \mathrm{MAD}=-64.4 \mathrm{~kJ} \mathrm{~mol}^{-1}$ (we note that these values remain essentially unchanged for the HF/CBS level of theory from W1-F12 and W1w theories). Second-order Møller-Plesset perturbation theory (MP2) systematically underestimates the reaction energies and results in an RMSD of $14.8 \mathrm{~kJ} \mathrm{~mol}^{-1}$ and an MSD $=-1 \times \mathrm{MAD}=-14.0 \mathrm{~kJ} \mathrm{~mol}^{-1}$. Nevertheless, it should be pointed out that MP2 still results in better performance compared to most of the DHDFT functionals (vide infra). Inclusion of higher-order excitations in the MP3 procedure improves upon the performance of MP2 and leads to an RMSD of $8.6 \mathrm{~kJ} \mathrm{~mol}^{-1}$. However, MP3 seems to overcorrect the deficiencies of MP2, and leads to systematic overestimation of the reaction energies (as is evident from MSD $=\mathrm{MAD}=7.6 \mathrm{~kJ} \mathrm{~mol}^{-1}$ ). In this situation, MP2.5, which is an average of MP2 and MP3, results in very good performance with an RMSD of $3.6 \mathrm{~kJ} \mathrm{~mol}^{-1}$ for the CR20 dataset. Fourth-order Møller-Plesset perturbation theory (MP4) provides a slight improvement over MP3, with an RMSD of $6.9 \mathrm{~kJ} \mathrm{~mol}^{-1}$. However, in contrast to MP3, MP4 systematically underestimates the reaction energies with $\mathrm{MSD}=-1 \times \mathrm{MAD}=-6.5 \mathrm{~kJ} \mathrm{~mol}^{-1}$. We note in passing, that similar oscillatory behavior of the MPn $(n=2,3$, and 4$)$ series has been previously observed for both thermochemical ${ }^{81,83,88,89,90}$ and kinetic properties. ${ }^{82,91}$ Since MP3 systematically overestimates the reaction energies and MP4 systematically underestimates them by similar amounts, MP3.5, which is an average of MP3 and MP4, results in excellent performance with an RMSD of only $2.4 \mathrm{~kJ} \mathrm{~mol}^{-1}$. In fact, this method outperforms all of the considered DHDFT and MPn-based procedures. The excellent performance of MP3.5 is also demonstrated by a relatively small MSD of $-1.6 \mathrm{~kJ} \mathrm{~mol}^{-1}$. Finally, it should be pointed out that scaling the same-spin and opposite-spin components of the MP2 and MP3 correlation energies, as in the SCS-MP2 and SCS-MP3 procedures, worsens the performance of both procedures and leads to RMSDs of over $20 \mathrm{~kJ} \mathrm{~mol}^{-1}$ (Table 3). This deterioration in performance upon scaling of the same-spin and opposite-spin components is rather unusual for reaction energies. ${ }^{92}$ For comparison, for the set of 140 atomization energies in the W4-11 dataset we obtain the following RMSDs for the MP $n$ and SCS-MP $n$ procedures: 45.8 (MP2), 28.1 (SCS-MP2), 60.5 (MP3), and $20.0 \mathrm{~kJ} \mathrm{~mol}^{-1}$ (SCS-MP3). ${ }^{25}$

The CCSD/A'VTZ level of theory shows excellent performance with an RMSD of $2.4 \mathrm{~kJ}$ $\mathrm{mol}^{-1}$. It should be noted, however, that at the CCSD/CBS level of theory (taken from W1-F12 
and $\mathrm{W} 1 \mathrm{w}$ theories) this RMSD increases to $4.1 \mathrm{~kJ} \mathrm{~mol}^{-1}$. The CCSD(T) method attains RMSDs of 8.7 and $4.7 \mathrm{~kJ} \mathrm{~mol}^{-1}$ in conjunction with the $\mathrm{A}^{\prime} \mathrm{VDZ}$ and $\mathrm{A}^{\prime} \mathrm{VTZ}$ basis sets, respectively. Therefore, it seems that the CCSD/A'VTZ level of theory benefits from some error compensation between basis set incompleteness and the neglect of quasiperturbative triples, $(\mathrm{T})$, corrections. It is of interest to assess the performance of the $\operatorname{CCSD}(\mathrm{T})$ method using an additivity-based approach in which the $\operatorname{CCSD}(\mathrm{T}) / \mathrm{CBS}$ energy is estimated from the $\mathrm{CCSD}(\mathrm{T}) / \mathrm{A}^{\prime} \mathrm{VDZ}$ energy and an MP2-based basis-set-correction term $\left(\triangle \mathrm{MP} 2=\mathrm{MP} 2 / \mathrm{A}^{\prime} \mathrm{V}\{\mathrm{T}, \mathrm{Q}\} \mathrm{Z}-\mathrm{MP} 2 / \mathrm{A}^{\prime} \mathrm{VDZ}\right.$, where the MP2/A'V $\{\mathrm{T}, \mathrm{Q}\} \mathrm{Z}$ energy is extrapolated to the basis-set limit with an extrapolation exponent of 3). ${ }^{93}$ This cost-effective approach, which is denoted here by $\operatorname{CCSD}(\mathrm{T}) / \mathrm{CBS}(\mathrm{MP} 2)$, has been widely used for obtaining noncovalent interaction energies close to the $\operatorname{CCSD}(\mathrm{T}) / \mathrm{CBS}$ limit. ${ }^{94,95,96,97}$ More recently, this method has also been found to give good performance for reaction energies ${ }^{89,90,98}$ and barrier heights. ${ }^{17,82}$ For the reactions in the CR20 database the $\mathrm{CCSD}(\mathrm{T}) / \mathrm{MP} 2(\mathrm{CBS})$ method gives an RMSD and MSD of $4.5 \mathrm{~kJ} \mathrm{~mol}^{-1}$, just above the threshold of chemical accuracy.

\subsection{Performance of DFT and DHDFT procedures for the reaction energies in the CR20}

database. Table 4 gives an overview of the performance of a large number of contemporary DFT and DHDFT functionals (with and without empirical D3 dispersion corrections). We start by making the following general observations:

$>$ The CR20 database proves to be an extremely challenging test for DFT methods. None of the 65 DFT and DHDFT functionals attain an RMSD below the threshold of chemical accuracy. Only 10 functionals attain RMSDs below $8.4 \mathrm{~kJ} \mathrm{~mol}^{-1}$ (twice the threshold of chemical accuracy). The rest of the functionals attain RMSDs ranging between 8.6 ( $\omega \mathrm{B} 97)$ and 60.9 (BLYP) $\mathrm{kJ} \mathrm{mol}^{-1}$.

$>$ Of the 53 conventional DFT methods (rungs 2-4 of Jacob's Ladder), nine functionals give RMSDs $<8.4 \mathrm{~kJ} \mathrm{~mol}^{-1}$. Of these, the range-separated hybrid GGA $\omega$ B97X functional of Chai and Head-Gordon gives the best performance with an RMSD of $4.7 \mathrm{~kJ} \mathrm{~mol}^{-1}$, whilst the hybrid GGA (B3PW91-D3) and the hybrid-meta GGA (M05-2X) tie for second place with RMSDs of 5.3 and $5.4 \mathrm{~kJ} \mathrm{~mol}^{-1}$, respectively.

$>$ Of the 12 considered double-hybrid functionals, only one functional (DSD-PBEP86-D3) gives an RMSD below $8.4 \mathrm{~kJ} \mathrm{~mol}^{-1}$. The other DHDFT functionals attain RMSDs between 13.6 (DSD-PBEP86) and 33.6 (B2-PLYP) $\mathrm{kJ} \mathrm{mol}^{-1}$. 
None of the considered DFT and DHDFT functionals result in largest deviations that are

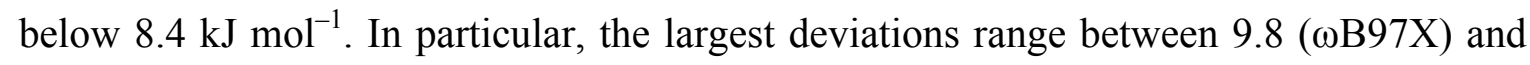
79.2 (BLYP) $\mathrm{kJ} \mathrm{mol}^{-1}$. We note that reactions 7 and 19 seem to be particularly problematic for most of the DFT procedures.

$>$ With few exceptions all the conventional and double-hybrid functionals tend to systematically underestimate the reaction energies, as evident from $\mathrm{MSD} \approx-1 \times \mathrm{MAD}$ (notable exceptions include $\omega \mathrm{B} 97, \mathrm{PBE} 0, \mathrm{LC}-\omega \mathrm{PBE}$, and M06-HF).

D Dispersion corrections tend to dramatically improve agreement with the W1-F12 and W1w results. For most of the functionals the RMSDs are reduced by $15-74 \%$ upon inclusion of the D3 dispersion corrections.

Table 4. Statistical analysis for the performance of DFT and DHDFT procedures for the calculation of the reaction energies in the CR20 database (in $\mathrm{kJ} \mathrm{mol}^{-1}$ ). ${ }^{a, b}$

\begin{tabular}{llcccc}
\hline Type $^{c}$ & Method & RMSD & MAD & MSD & LD \\
\hline GGA & BLYP & 60.9 & 60.3 & -60.3 & $79.2(\mathbf{1 9})$ \\
& BLYP-D3 & 38.9 & 38.4 & -38.4 & $48.7(7)$ \\
& B97-D & 52.4 & 52.2 & -52.2 & $59.6(\mathbf{1 7})$ \\
& HCTH407 & 54.4 & 53.6 & -53.6 & $69.5(\mathbf{1 9})$ \\
& PBE & 11.1 & 9.0 & -8.0 & $21.4(\mathbf{9})$ \\
& PBE-D3 & 7.6 & 6.4 & 2.4 & $18.6(\mathbf{2 0})$ \\
& BP86 & 21.7 & 20.3 & -20.3 & $34.4(\mathbf{1 9})$ \\
& BP86-D3 & 7.7 & 5.8 & -3.5 & $15.3(\mathbf{7})$ \\
& BPW91 & 31.1 & 30.0 & -30.0 & $45.2(\mathbf{9})$ \\
& SOGGA11 & 29.1 & 26.8 & -26.8 & $48.3(\mathbf{7})$ \\
& N12 & 22.8 & 20.7 & -20.7 & $37.7(\mathbf{1 9})$ \\
MGGA & M06-L & 49.9 & 49.6 & -49.6 & $58.4(7)$ \\
& TPSS & 21.6 & 19.7 & -19.6 & $36.6(\mathbf{1 9})$ \\
& TPSS-D3 & 9.2 & 8.2 & -6.4 & $15.5(\mathbf{7})$ \\
& -HCTH & 49.8 & 48.5 & -48.5 & $69.5(\mathbf{9})$ \\
& VSXC & 47.5 & 47.2 & -47.2 & $59.9(7)$ \\
& BB95 & 36.8 & 35.8 & -35.8 & $50.1(\mathbf{1 9})$ \\
& M11-L & 36.9 & 36.4 & -36.4 & $46.4(\mathbf{1 1})$ \\
& MN12-L & 15.7 & 12.7 & -10.9 & $30.4(\mathbf{1 7})$ \\
HGGA & BH\&HLYP & 28.9 & 27.6 & -27.6 & $47.1(\mathbf{1 9})$ \\
& BH\&HLYP-D3 & 14.3 & 13.6 & -13.6 & $22.7(\mathbf{1 9})$ \\
& B3LYP & 43.2 & 42.5 & -42.5 & $61.3(\mathbf{1 9})$ \\
& B3LYP-D3 & 24.9 & 24.5 & -24.5 & $31.6(\mathbf{7})$ \\
& B3P86 & 9.7 & 7.4 & -7.0 & $21.8(\mathbf{1 9})$ \\
& B3PW91 & 20.2 & 18.9 & -18.9 & $34.3(\mathbf{1 9})$ \\
& B3PW91-D3 & 5.3 & 4.2 & -0.6 & $10.7(\mathbf{7})$ \\
& PBE0 & 6.7 & 5.9 & 2.9 & $10.7(\mathbf{1 1})$ \\
& PBE0-D3 & 13.0 & 12.1 & 12.1 & $18.9(\mathbf{2 0})$ \\
& B97-1 & 18.8 & 17.7 & -17.7 & $31.9(\mathbf{1 9})$ \\
\hline
\end{tabular}




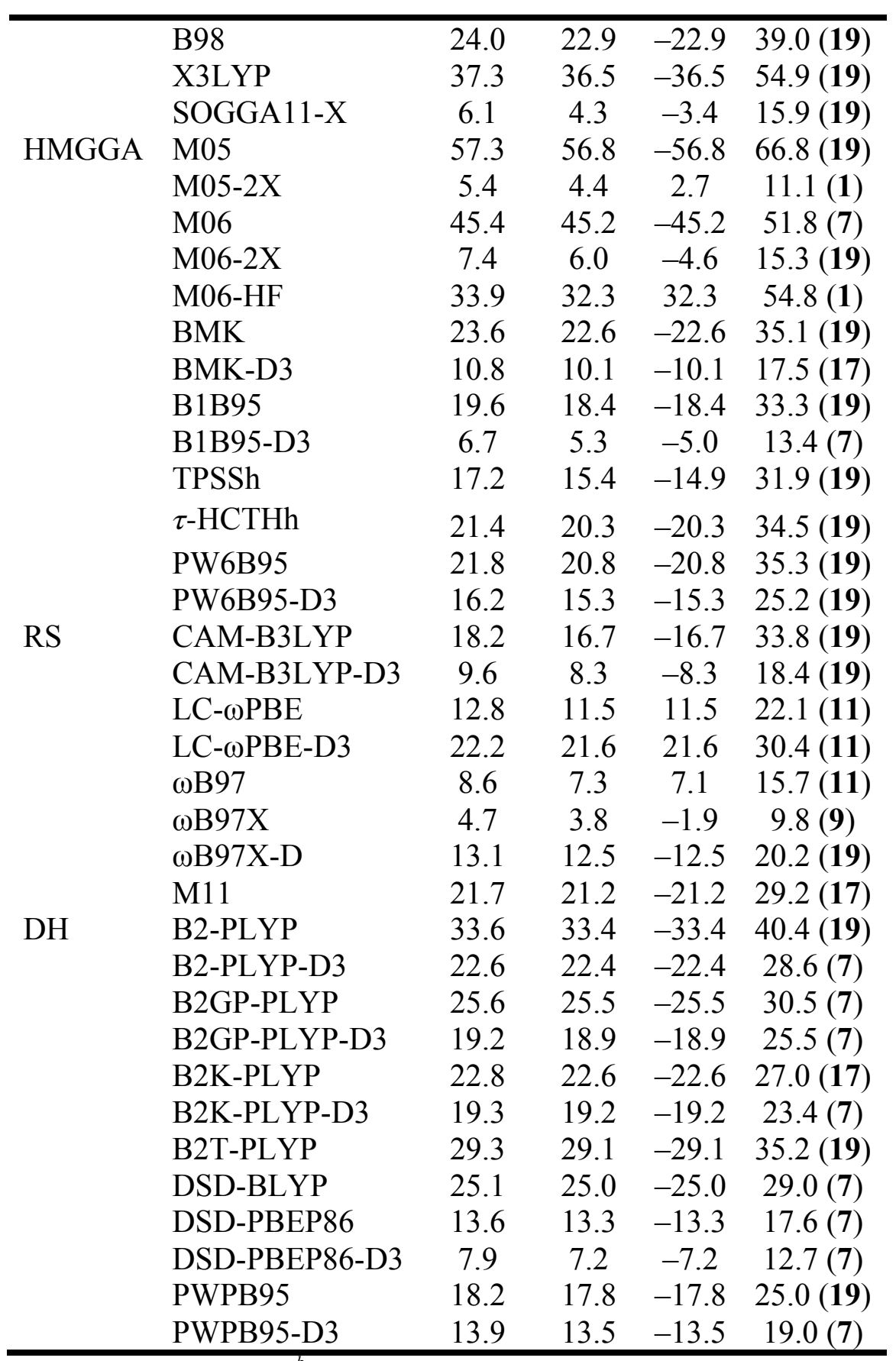

${ }^{a}$ Footnotes $a$ and $b$ to Table 3 apply here. ${ }^{b}$ The standard DFT calculations are carried out in conjunction with the $\mathrm{A}^{\prime} \mathrm{VTZ}$ basis set while the DHDFT calculations are carried out in conjunction with the $\mathrm{A}^{\prime} \mathrm{VQZ}$ basis set. ${ }^{c} \mathrm{GGA}=$ generalized gradient approximation, $\mathrm{HGGA}=$ hybrid-GGA, MGGA = meta-GGA, RS = range-separated, HMGGA = hybrid-meta-GGA, $\mathrm{DH}=$ double hybrid.

Two of the eleven considered GGA functionals give relatively good performance with RMSDs below the $8.4 \mathrm{~kJ} \mathrm{~mol}^{-1}$ threshold $\left(\mathrm{RMSD}=7.6(\mathrm{PBE}-\mathrm{D} 3)\right.$ and $\left.7.7(\mathrm{BP} 86-\mathrm{D} 3) \mathrm{kJ} \mathrm{mol}^{-1}\right)$. We note that removing the D3 dispersion correction significantly increases the RMSDs for these two functionals, namely to 11.1 (PBE) and 21.7 (BP86) $\mathrm{kJ} \mathrm{mol}^{-1}$. The rest of the GGAs give very poor performance with RMSDs between 22.8 (N12) and 60.9 (BLYP) kJ mol ${ }^{-1}$. 
Inclusion of the kinetic energy density in the MGGA procedures does not improve the performance. The best performing MGGA functional attains an RMSD of $9.2 \mathrm{~kJ} \mathrm{~mol}^{-1}$ (TPSSD3), we note that removing the D3 dispersion correction significantly increases the RMSD by $12.4 \mathrm{~kJ} \mathrm{~mol}^{-1}$. With the exception of PBE-D3, all the GGA and MGGA functionals systematically underestimate the reactions energies as evident from MSD $\approx-1 \times$ MAD. PBE-D3 attains a slightly positive MSD of $+2.4 \mathrm{~kJ} \mathrm{~mol}^{-1}$ (cf. with an MSD of $-8.0 \mathrm{~kJ} \mathrm{~mol}^{-1}$ for PBE). These results suggest that, apart from PBE-D3 and BP86-D3, the GGA and MGGA functionals should be applied with caution when calculating cycloreversion reaction energies.

The RMSDs for the HGGA functionals span a wide range, from 5.3 (B3PW91-D3) to 43.2 (B3LYP) $\mathrm{kJ} \mathrm{mol}^{-1}$. Of the 13 considered hybrid GGAs, three functionals result in RMSDs $<8.4$ $\mathrm{kJ} \mathrm{mol}^{-1}$, these are: B3PW91-D3 (5.3), SOGGA11-X (6.1), and PBE0 (6.7 kJ mol $\left.{ }^{-1}\right)$. We note however, that the RMSD for B3PW91-D3 increases to as much as $20.2 \mathrm{~kJ} \mathrm{~mol}^{-1}$ upon removal of the D3 dispersion correction. We also note that the popular B3LYP functional shows very poor performance with RMSDs of 24.9 and 43.2 and $\mathrm{kJ} \mathrm{mol}^{-1}$ with and without the D3 dispersion correction, respectively. It is instructive to compare the performance of the hybrid functionals that combine Becke's three-parameter exchange functional with different gradient-corrected correlation functionals. These functionals give RMSDs of 9.7 (B3P86), 20.2 (B3PW91), and 43.2 (B3LYP) $\mathrm{kJ} \mathrm{mol}^{-1}$. This reflects the poor performance of the LYP and PW91 correlation functionals for these reactions (see also the discussion of the performance of the DHDFT functionals below). Similar observations have been also noted for other types of reactions, such as bond dissociation energies of $\mathrm{N}-\mathrm{H}$ and $\mathrm{N}-\mathrm{Cl}$ bonds. ${ }^{99}$

In general, the HMGGA functionals give similar performance to the HGGAs, with a wide spread of RMSDs. Interestingly, both the best and worst performing HMGGA functionals belong to the Minnesota-05 family, with RMSDs of 5.4 (M05-2X) to 57.3 (M05) kJ mol ${ }^{-1}$. Three out of the 13 considered HMGGA functionals result in RMSDs $<8.4 \mathrm{~kJ} \mathrm{~mol}^{-1}$, namely M05-2X (5.4), B1B95-D3 (6.7), and M06-2X (7.4 kJ mol $\left.{ }^{-1}\right)$. We note that the RMSD for B1B95-D3 increases to as much as $19.6 \mathrm{~kJ} \mathrm{~mol}^{-1}$ upon removal of the $\mathrm{D} 3$ dispersion correction.

Inspection of Table 4 reveals that the optimal percentage of HF-like exchange for the HGGA and HMGGA functionals spans over a wide range of 20-55\%. For example, the best performing functionals with $20-28 \%$ of HF-like exchange are (RMSDs are given in parenthesis): B3P86 (7.3), PBE0-D3 (5.8), PBE0 (6.2), and B1B95-D3 (7.7 kJ mol ${ }^{-1}$ ). At the other end, functionals with $40-55 \%$ of HF-like exchange that perform well are: SOGGA11-X (5.6), BH\&HLYP-D3 (7.2), and M06-2X $\left(6.8 \mathrm{~kJ} \mathrm{~mol}^{-1}\right)$. We note that similar observations have been made for the barrier heights of the reactions in the $\mathrm{CRBH} 20$ dataset. $^{17}$ 
The RMSDs for the range-separated hybrid- and hybrid-meta-GGAs range between 4.7 $(\omega \mathrm{B} 97 \mathrm{X})$ and 22.2 (LC- $\omega$ PBE-D3) $\mathrm{kJ} \mathrm{mol}^{-1}$, where $\omega$ B97X clearly outperforms the rest of the functionals. The good performance of $\omega \mathrm{B} 97 \mathrm{X}$ is followed by $\omega \mathrm{B} 97$ (RMSD = 8.6) and CAMB3LYP-D3 $\left(\right.$ RMSD $\left.=9.6 \mathrm{~kJ} \mathrm{~mol}^{-1}\right)$.

All of the considered double-hybrid functionals lead to disappointing RMSDs considering their high computational cost. In particular, the RMSDs for the DHDFT procedures range between 7.9 (DSD-PBEP86-D3) and 33.6 (B2-PLYP) $\mathrm{kJ} \mathrm{mol}^{-1}$. We note that the DHDFT functionals that employ the LYP correlation functional give poor performance with RMSDs ranging between 19.2 (B2GP-PLYP-D3) and 33.6 (B2-PLYP) kJ mol ${ }^{-1}$. Notably, these DHDFT procedures perform significantly worse than MP2 (which attains an RMSD of $14.8 \mathrm{~kJ} \mathrm{~mol}^{-1}$, Table 3). The spin-component-scaled DHDFT procedures (PWPB95 and DSD-PBEP86) give better performance, but still perform on par or worse than MP2, with RMSDs of 18.2 and $13.6 \mathrm{~kJ}$ $\mathrm{mol}^{-1}$, respectively. Finally, we note that dispersion corrections reduce the RMSDs of the DHDFT functionals by considerable amounts; however, DSD-PBEP86-D3 (RMSD $=7.8 \mathrm{~kJ} \mathrm{~mol}^{-}$ ${ }^{1}$ ) is the only DHDFT functional that manages to slightly surpass the RMSD cutoff value of 8.4 $\mathrm{kJ} \mathrm{mol}^{-1}$.

As mentioned above, without dispersion corrections nearly all the conventional and doublehybrid functionals tend to systematically underestimate the reaction energies, as evident from $\mathrm{MSD} \approx-1 \times$ MAD. Dispersion effects make the reaction energies less exothermic since they stabilize the heterocyclic rings are to a larger extent than the products of the cycloreversion reaction (Fig. 1). Therefore, dispersion D3 corrections tend to systematically improve the performance of the DFT and DHDFT methods. For the few functionals that tend to overestimate the reaction energies (e.g., PBE0 and LC- $\omega$ PBE) there is no point in including D3 corrections since they can only increase the errors. Table 5 gathers the differences in RMSD between the dispersion-corrected and uncorrected DFT functionals $(\triangle \mathrm{RMSD}=\mathrm{RMSD}(\mathrm{DFT})-\mathrm{RMSD}(\mathrm{DFT}$ D3)). A positive $\triangle \mathrm{RMSD}$ value indicates that the dispersion correction improves the performance of the functional, whereas a negative $\triangle \mathrm{RMSD}$ value indicates deterioration in performance. Inspection of Table 5 reveals that, with few exceptions, the dispersion D3 corrections significantly improve the agreement with the W1-F12 and W1w results. In particular, the RMSDs are reduced by amounts ranging from 3.5 (PBE) and 22.0 (BLYP) kJ mol ${ }^{-1}$ upon inclusion of the dispersion correction. Cases where the RMSD is reduced by more than $50 \%$ upon inclusion of the D3 dispersion correction include: BH\&HLYP, BMK, TPSS, BP86, B1B95, and B3PW91. Finally, it is worth pointing out that sizable dispersion corrections are still obtained for the 
DHDFT functionals, in particular, the RMSDs are reduced by amounts ranging between 3.5 (B2K-PLYP) and 11.0 (B2-PLYP) $\mathrm{kJ} \mathrm{mol}^{-1}$.

Table 5. Overview of the performance of various DFT functionals with and without empirical D3 dispersion corrections. The tabulated values are $\triangle \mathrm{RMSD}=\mathrm{RMSD}(\mathrm{DFT})-\mathrm{RMSD}(\mathrm{DFT}-\mathrm{D} 3$ ) (in $\left.\mathrm{kJ} \mathrm{mol}^{-1}\right){ }^{a}$

\begin{tabular}{llc}
\hline Type & Method & $\Delta$ RMSD \\
\hline GGA & BLYP & 22.0 \\
& PBE & 3.5 \\
& BP86 & 14.0 \\
MGGA & TPSS & 12.4 \\
HGGA & BH\&HLYP & 14.6 \\
& B3LYP & 18.3 \\
& B3PW91 & 14.9 \\
& PBE0 & -6.3 \\
HMGGA & BMK & 12.8 \\
& B1B95 & 12.9 \\
& PW6B95 & 5.6 \\
RS & CAM-B3LYP & 8.6 \\
& LC- $\omega$ PBE & -9.4 \\
& कB97X & -8.4 \\
DH & B2-PLYP & 11.0 \\
& B2GP-PLYP & 6.4 \\
& B2K-PLYP & 3.5 \\
& DSD-PBEP86 & 5.7 \\
& PWPB95 & 4.3 \\
\hline
\end{tabular}

${ }^{a}$ Footnotes $a-c$ to Table 4 apply here. ${ }^{b}$ The original dispersion correction rather than the D3 correction is used for this functional.

It is generally accepted that the accuracy of DFT methods should increase as one climbs the rungs of Jacob's Ladder. One way of demonstrating this is to take the average of the RMSDs obtained for the DFT functionals from each rung of Jacob's Ladder. Let us consider, for example, the performance of DFT for the atomization energies in the W4-11 dataset. Reference 25 reports the RMSDs for a wide range of functionals from rungs 2-5 of Jacob's Ladder relative to highly accurate reference values from W4 (or higher) theory. ${ }^{19}$ Taking the average of the RMSDs obtained for the functionals from each rung of Jacob's Ladder we obtain: 37.8 (pure GGAs, rung two), 21.9 (MGGAs, rung three), 16.1 (HMGGAs, rung four), and 9.1 (DHDFT, rung five) kJ $\mathrm{mol}^{-1}$. Thus, the averaged RMSD is reduced as one climbs the rungs of Jacob's Ladder. In contrast, for the reactions in the CR20 dataset we obtain the following averaged RMSDs: 30.7 (over 11 pure GGAs), 33.4 (over 8 MGGAs), 22.1 (over 13 HMGGAs), and 20.9 (over 12 DHDFT) $\mathrm{kJ} \mathrm{mol}^{-1}$. Thus, there is little or no improvement when going from rungs $2 \rightarrow 3$ and 4 $\rightarrow 5$. Having said that, we note that taking the average RMSD over a set of $\sim 10$ DFT functionals 
from each rung of Jacob's Ladder is a crude approach for differentiating between the performance of the different rungs. Thus, it is useful to also compare the RMSD of the best performer from each rung rather than the averaged RMSD. The RMSD of the best performers are: 7.6 (GGA), 9.2 (MGGA), 5.4 (HMGGA), and 7.9 (DHDFT) $\mathrm{kJ} \mathrm{mol}^{-1}$. Again, we see that there is deterioration in performance from rungs $2 \rightarrow 3$ and $4 \rightarrow 5$.

\subsection{Performance of theoretical procedures for both reaction energies and barrier heights.}

In order to carry out computational investigations of the mechanisms of cycloreversion reactions we need to identify theoretical procedures that can accurately describe all aspects of the potential energy surface (i.e., give both reliable reaction energies and barrier heights). This is particularly important for investigations, such as Ref. 14, that consider a number of competing reaction pathways which involve reaction energies and barrier heights that are separated by only a few kJ $\mathrm{mol}^{-1}$. The results presented here for reaction energies and in Ref. 17 for reaction barrier heights reveal a frustrating situation in which it is hard to find theoretical procedures that perform reasonably well for both reaction energies and barrier heights. This section identifies the best DFT and ab initio methods that are recommended for investigating the entire potential energy surface of cycloreversion reactions.

First, let us consider the performance of the DFT methods. None of the considered DFT and DHDFT methods are able to calculate the reaction energies with RMSDs below the chemical accuracy threshold (Table 4). Thus, we will point out the procedures that result in RMSDs below the threshold of twice that of chemical accuracy (i.e., RMSD $\leq 8.4 \mathrm{~kJ} \mathrm{~mol}^{-1}$ ). In Section 3.3 we identified 10 functionals that can achieve this goal. Namely, the GGAs: PBE-D3 and BP86-D3; the HGGAs B3PW91-D3, SOGGA11-X, and PBE0; the HMGGAs M05-2X, B1B95-D3, and M06-2X; and the DHDFT DSD-PBEP86-D3. As may be expected, both GGA functionals significantly underestimate the reaction barrier heights (with RMSDs of over $30 \mathrm{~kJ} \mathrm{~mol}^{-1}$ for the CRBH20 dataset). ${ }^{17}$ The HGGA B3PW91-D3 and the HMGGA M05-2X also result in relatively poor performance for the reaction barrier heights (with RMSDs of over $10 \mathrm{~kJ} \mathrm{~mol}^{-1}$ ). ${ }^{17} \mathrm{We}$ therefore do not recommend the use of these four functionals for the calculation of both the energetics and kinetics of cycloreversion reaction profiles. Table 6 gathers the RMSDs for the CR20 and CRBH20 databases for the rest of the best performing functionals. Looking at the average of the RMSDs for both the CR20 and CRBH20 databases, the DHDFT DSD-PBEP86D3 method emerges as the best performer with an averaged RMSD of $5.2 \mathrm{~kJ} \mathrm{~mol}^{-1}$. However, whilst this method gives superb performance for the reaction barrier heights $(\mathrm{RMSD}=2.5 \mathrm{~kJ}$ $\mathrm{mol}^{-1}$ ), it shows relatively poor performance for the reaction energies $\left(\mathrm{RMSD}=7.9 \mathrm{~kJ} \mathrm{~mol}^{-1}\right.$ ). 
The HGGA SOGGA11-X and the range-separated HGGA $\omega$ B97X emerge as the second best performers with averaged RMSDs of 5.9-6.0 $\mathrm{kJ} \mathrm{mol}^{-1}$. Of these functionals, SOGGA11-X gives slightly better performance for the reaction barrier heights, whereas $\omega$ B97X gives slightly better performance for the reaction energies. Overall, both of these functionals are equally recommended for investigations of the mechanisms of cycloreversion reactions.

Table 6. Performance of DFT and ab initio methods for both the reaction energies (CR20 dataset) and barrier heights (CRBH20 dataset) of cycloreversion reactions (in $\mathrm{kJ} \mathrm{mol}^{-1}$ ).

\begin{tabular}{|c|c|c|c|}
\hline Methods & $\mathrm{CR} 20^{a}$ & $\mathrm{CRBH} 20^{a}$ & Both datasets $^{b}$ \\
\hline \multicolumn{4}{|c|}{ DFT and DHDFT methods ${ }^{c}$} \\
\hline SOGGA11-X & 6.1 & 5.6 & 5.9 \\
\hline PBE0 & 6.7 & 6.2 & 6.5 \\
\hline B1B95-D3 & 6.7 & 7.7 & 7.2 \\
\hline M06-2X & 7.4 & 6.8 & 7.1 \\
\hline$\omega \mathrm{B} 97 \mathrm{X}$ & 4.7 & 7.3 & 6.0 \\
\hline DSD-PBEP86-D3 & 7.9 & 2.5 & 5.2 \\
\hline \multicolumn{4}{|c|}{ Composite ab initio methods ${ }^{d}$} \\
\hline CBS-QB3 & 2.9 & 3.1 & 3.0 \\
\hline CBS-APNO ${ }^{e}$ & 3.4 & 1.5 & 2.5 \\
\hline \multicolumn{4}{|c|}{ Standard ab initio methods ${ }^{f}$} \\
\hline $\operatorname{CCSD}(\mathrm{T}) / \mathrm{A}^{\prime} \mathrm{VTZ}$ & 4.7 & 5.0 & 4.9 \\
\hline $\mathrm{CCSD}(\mathrm{T}) / \mathrm{CBS}(\mathrm{MP} 2)^{g}$ & 4.5 & 1.7 & 3.1 \\
\hline
\end{tabular}

${ }^{a}$ RMSDs for the CR20 and CRBH20 datasets. ${ }^{b}$ Average of the RMSD for the CR20 and CRBH20 datasets. ${ }^{c}$ DFT methods with RMSD $<8.4 \mathrm{~kJ} \mathrm{~mol}^{-1}$ for both the CR20 and CRBH20 datasets. ${ }^{d}$ Composite ab initio methods with RMSD $<4.2 \mathrm{~kJ} \mathrm{~mol}^{-1}$ for both the CR20 and CRBH20 datasets. ${ }^{e}$ Error statistics over the dioxazole systems only. ${ }^{f}$ Standard ab initio methods with RMSD $\leq 5.0 \mathrm{~kJ} \mathrm{~mol}^{-1}$ for both the CR20 and CRBH20 datasets. ${ }^{g} \mathrm{CCSD}(\mathrm{T}) / \mathrm{CBS}(\mathrm{MP} 2) \approx \mathrm{CCSD}(\mathrm{T}) / \mathrm{A}^{\prime} \mathrm{VDZ}+\mathrm{MP} 2 / \mathrm{A}^{\prime} \mathrm{V}\{\mathrm{T}, \mathrm{Q}\} \mathrm{Z}-\mathrm{MP} 2 / \mathrm{A}^{\prime} \mathrm{VDZ}$.

Let us now move to the performance of the composite ab initio methods. In Section 3.2 we identified five methods that are able to calculate the reaction energies with RMSDs below the chemical accuracy threshold (Table 3). Namely, CBS-QB3, CBS-APNO G3, G4, and G4(MP2)6X. Of these, the Gn methods result in RMSDs above the threshold of chemical accuracy for the reaction barrier heights (namely, $\mathrm{RMSD}=4.9-6.4 \mathrm{~kJ} \mathrm{~mol}^{-1}$ ). However, the CBS-type methods result in excellent performance for both the reaction energies and barrier heights, with RMSDs well below the threshold of chemical accuracy. Whereas CBS-APNO gives significantly better performance for the reaction barrier heights, CBS-QB3 gives better performance for the reaction energies. Overall, both methods are recommended for investigations of the potential energy surfaces of cycloreversion reactions.

Finally, let us consider the performance of the standard ab initio methods. In Section 3.2 we identified three methods with RMSDs below the chemical accuracy threshold for the CR20 
dataset (namely, MP2.5/A'VQZ, MP3.5/A'VTZ, and CCSD/A'VTZ). Of these, MP2.5 and MP3.5 exhibit particularly poor performance for the barrier heights, with RMSDs of 26.4 and $11.3 \mathrm{~kJ}$ $\mathrm{mol}^{-1}$, respectively. The CCSD/A'VTZ also results in an unsatisfactory RMSD of $6.3 \mathrm{~kJ} \mathrm{~mol}^{-1}$, for the CRBH20 database. Table 6 lists the standard ab initio methods that result in RMSDs $\leq 5.0$ $\mathrm{kJ} \mathrm{mol}^{-1}$ for both the CR20 and CRBH20 datasets. The CCSD(T)/A'VTZ level of theory just achieves this goal with RMSDs of 4.7 and 5.0, $\mathrm{kJ} \mathrm{mol}^{-1}$, respectively. The MP2-based additivity approach for estimating the $\operatorname{CCSD}(\mathrm{T}) / \mathrm{CBS}$ energy (CCSD(T)/CBS(MP2)) shows similar performance for the reaction energies, but significantly improved performance for the reaction barrier heights. We therefore recommend the $\operatorname{CCSD}(\mathrm{T}) / \mathrm{CBS}(\mathrm{MP} 2)$ method for investigating the potential energy surface of cycloreversion reactions.

\section{Conclusions}

We introduce a representative benchmark database of 20 cycloreversion reaction energies (to be known as the CR20 dataset). The reference reaction energies are obtained at the $\operatorname{CCSD}(\mathrm{T}) / \mathrm{CBS}$ level by means of the high-level W1-F12 and W1w thermochemical protocols. These benchmark values allow us to assess the performance of more approximate theoretical procedures. Specifically, we examine the performance of a variety of contemporary DFT and DHDFT procedures, as well as a number of standard and composite ab initio methods. With regard to the performance of the DFT and DHDFT procedures we make the following observations:

$>$ The CR20 dataset proves to be an extremely challenging test for all of the considered DFT and DHDFT procedures (rungs 2-5 of Jacob's Ladder). With no exceptions, all the DFT and DHDFT methods result in RMSDs above the 'chemical accuracy' threshold (i.e., RMSDs $>4.2 \mathrm{~kJ} \mathrm{~mol}^{-1}$ ). Increasing the threshold of an acceptable RMSD to twice that of chemical accuracy (i.e., RMSD $=8.4 \mathrm{~kJ} \mathrm{~mol}^{-1}$ ), results in 10 functionals with acceptable performance.

$>$ The best performing DFT functionals (RMSDs are given in parenthesis) are the GGAs: PBE-D3 (7.6) and BP86-D3 (7.7); the HGGAs B3PW91-D3 (5.3), SOGGA11-X (6.1), and PBE0 (6.7); the HMGGAs M05-2X (5.4), B1B95-D3 (6.7), and M06-2X (7.4); and the DHDFT DSD-PBEP86-D3 $\left(7.9 \mathrm{~kJ} \mathrm{~mol}^{-1}\right)$.

$>$ The rest of the functionals attain RMSDs ranging between $8.6(\omega \mathrm{B} 97)$ and 60.9 (BLYP) $\mathrm{kJ} \mathrm{mol}^{-1}$. 
Empirical D3 dispersion corrections significantly improve the performance of most of the DFT functionals and are essential for obtaining reasonable performance for the CR20 dataset. In particular, the RMSDs for most of the functionals are reduced by $15-74 \%$ upon inclusion of the D3 dispersion corrections.

With regard to the performance of the standard and composite ab initio procedures, we draw the following conclusions:

$>$ The composite ab initio procedures show good-to-excellent performance for the CR20 dataset. The best performing composite procedures with RMSDs below the threshold of chemical accuracy are: CBS-QB3 (2.9), CBS-APNO (3.4), G4(MP2)-6X (3.8), G3 and $\mathrm{G} 4\left(4.1 \mathrm{~kJ} \mathrm{~mol}^{-1}\right)$.

$>$ The best performing MPn-based procedures with RMSDs below the threshold of chemical accuracy are: MP2.5 (3.6) and MP3.5 (2.4 kJ mol$\left.{ }^{-1}\right)$. The rest of the MPn-based procedures show relatively poor performance with RMSDs between 4.8 (MP4(SDQ)) and 23.4 (SCS-MP2) $\mathrm{kJ} \mathrm{mol}^{-1}$.

$>$ The $\operatorname{CCSD}(\mathrm{T})$ method in conjunction with the $\mathrm{A}^{\prime} \mathrm{VDZ}$ and $\mathrm{A}^{\prime} \mathrm{VTZ}$ basis sets gives RMSDs of 8.7 and $4.7 \mathrm{~kJ} \mathrm{~mol}^{-1}$, respectively. Estimating the CCSD(T)/CBS energy from the $\operatorname{CCSD}(\mathrm{T}) / \mathrm{A}^{\prime} \mathrm{VDZ}$ energy and adding an MP2-based basis-set-correction term (i.e., the $\mathrm{CCSD}(\mathrm{T}) / \mathrm{CBS}(\mathrm{MP} 2)$ method) results in an RMSD of $4.5 \mathrm{~kJ} \mathrm{~mol}^{-1}$.

Based on the results presented here for reaction energies and in Ref. 17 for reaction barrier heights we find that only a handful of DFT and ab initio methods are suitable for investigating the entire potential energy surface of cycloreversion reactions. In particular, we recommend the following methods for such investigations:

$>$ Of the DFT methods, the HGGA SOGGA11-X and the range-separated HGGA $\omega$ B97X emerge as the best performers with RMSDs between 4.7-7.3 kJ mol${ }^{-1}$, for the reaction energies and barrier heights.

$>$ Of the DHDFT methods, DSD-PBEP86-D3 shows the best performance with RMSDs of 2.5 and $7.9 \mathrm{~kJ} \mathrm{~mol}^{-1}$ for the CRBH20 and CR20 databases, respectively. 
$>$ Of the standard ab initio methods, the $\operatorname{CCSD}(\mathrm{T}) / \mathrm{CBS}(\mathrm{MP} 2)$ method emerges as the best performer with RMSDs of 1.7 and $4.5 \mathrm{~kJ} \mathrm{~mol}^{-1}$ for the CRBH20 and CR20 databases, respectively.

Of the composite methods, CBS-APNO and CBS-QB3 give excellent performance for both the reaction energies and barrier heights, with RMSDs between 1.5-3.4 $\mathrm{kJ} \mathrm{mol}^{-1}$. Overall, the CBS-type procedures are the methods of choice for investigating the entire potential energy surface of cycloreversion reactions.

\section{Appendix A. Supplementary data}

Diagnostics indicating the importance of post-CCSD $(\mathrm{T})$ contributions for the species involved in the CR20 database (Table S1); overview of the basis set convergence of the standard and modified ab initio procedures (Table S2); overview of the basis set convergence of conventional DFT methods (Table S3); and full references for Ref. 21 (Molpro 2012), Ref. 33 (Gaussian 09), and Ref. 73 (ORCA). For the convenience of the reader the Supplementary data also includes a directory (CR20_input_files_and_script.zip) with the Gaussian 09 input files for the species involved in the CR20 database and a perl script that calculates the reaction energies and the error statistics from our W1-F12 and W1w reference values.

\section{Acknowledgments}

This research was undertaken with the assistance of resources from the National Computational Infrastructure (NCI), which is supported by the Australian Government. We gratefully acknowledge the system administration support provided by the Faculty of Science at UWA to the Linux cluster of the Karton group, the provision of an Australian Postgraduate Award (to LJ.Y.), and an Australian Research Council (ARC) Discovery Early Career Researcher Award (to A.K., project number: DE140100311).

\section{References}

${ }^{1}$ J. P. Perdew, and K. Schmidt, AIP Conf. Proc. 577, 1 (2001).

2 J. Baker, J. Andzelm, M. Muir, and P. R. Taylor, Chem. Phys. Lett. 237, 53 (1995).

3 J. L. Durant, Chem. Phys. Lett. 256, 595 (1996).

${ }^{4}$ A. D. Boese, J. M. L. Martin, and N. C. Handy, J. Chem. Phys. 119, 3005 (2003).

${ }^{5}$ A. D. Boese, and J. M. L. Martin, J. Chem. Phys. 121, 3405 (2004).

${ }^{6}$ A. J. Cohen, P. Mori-Sánchez, and W. Yang, Chem. Rev. 112, 289 (2012).

${ }^{7}$ B. J. Lynch, P. L. Fast, M. Harris, and D. G. Truhlar, J. Phys. Chem. A 104, 4811 (2000). 
${ }^{8}$ J. K. Kang, and C. B. Musgrave, J. Chem. Phys. 115, 11040 (2001).

${ }^{9}$ Y. Zhao, and D. G. Truhlar, Theor. Chem. Acc. 120, 215 (2008).

${ }^{10}$ A. Karton, A. Tarnopolsky, J.-F. Lamere, G. C. Schatz, and J. M. L. Martin, J. Phys. Chem. A 112, 12868 (2008).

${ }^{11}$ S. Kozuch, and J. M. L. Martin, J. Comp. Chem. 34, 2327 (2013).

${ }^{12}$ L. Goerigk, S. Grimme, WIREs Comput. Mol. Sci. 4, 576 (2014).

${ }^{13}$ B. A. Burkett, J. M. Kane-Barber, R. J. O’Reilly, and L. Shi, Tetrahedron Lett. 48, 5355 (2007).

${ }^{14}$ R. J. O’Reilly, and L. Radom, Org. Lett. 11, 1325 (2009).

${ }^{15}$ J. N. Kim, K. S. Jung, H. J. Lee, and J. S. Son, Tetrahedron Lett. 38, 1596 (1997).

${ }^{16}$ Y. Hu, C.-Y. Li, X.-M. Wang, Y.-H. Yang, and H.-L. Zhu, Chem. Rev. 114, 5572 (2014).

${ }^{17}$ L.-J. Yu, F. Sarrami, R. J. O’Reilly, and A. Karton, Chem. Phys. 458, 1 (2015). DOI:

10.1016/j.chemphys.2015.07.005

${ }^{18}$ J. M. L. Martin, and G. Oliveira, J. Chem. Phys. 111, 1843 (1999).

${ }^{19}$ A. Karton, E. Rabinovich, J. M. L. Martin, and B. Ruscic, J. Chem. Phys. 125, 144108 (2006).

${ }^{20}$ A. Karton, and J. M. L. Martin, J. Chem. Phys. 136, 124114 (2012).

${ }^{21}$ MOLPRO is a package of ab initio programs written by H.-J. Werner, P. J. Knowles, G.

Knizia, F. R. Manby, M. Schütz, P. Celani, T. Korona, R. Lindh, A. Mitrushenkov, G. Rauhut, et al. See: http:www.molpro.net.

${ }^{22}$ H.-J. Werner, P. J. Knowles, G. Knizia, F. R. Manby, and M. Schütz, WIREs Comput. Mol. Sci. 2, 242 (2012).

${ }^{23}$ K. A. Peterson, D. Feller, and D. A. Dixon, Theor. Chem. Acc. 131, 1079 (2012).

${ }^{24}$ T. Helgaker, W. Klopper, and D. P. Tew, Mol. Phys. 106, 2107 (2008).

${ }^{25}$ A. Karton, S. Daon, and J. M. L. Martin, Chem. Phys. Lett. 510, 165 (2011).

${ }^{26}$ T. H. Dunning, J. Chem. Phys. 90, 1007 (1989).

${ }^{27}$ R. A. Kendall, T. H. Dunning, Jr., and R. J. Harrison, J. Chem. Phys. 96, 6796 (1992).

${ }^{28}$ T. H. Dunning, K. A. Peterson, and A. K. Wilson, J. Chem. Phys. 114, 9244 (2001).

${ }^{29}$ A. Karton, Chem. Phys. Lett. 585, 330 (2014).

${ }^{30}$ C. Lee, W. Yang, and R. G. Parr, Phys. Rev. B 37, 785 (1988).

${ }^{31}$ A. D. Becke, J. Chem. Phys. 98, 5648 (1993).

${ }^{32}$ P. J. Stephens, F. J. Devlin, C. F. Chabalowski, and M. J. Frisch, J. Phys. Chem. 98, 11623 (1994). 
${ }^{33}$ M. J. Frisch, G. W. Trucks, H. B. Schlegel, G. E. Scuseria, M. A. Robb, J. R. Cheeseman, G. Scalmani, V. Barone, B. Mennucci, G. A. Petersson, et al. Gaussian 09, Revision D.01; Gaussian, Inc.: Wallingford CT, 2009.

${ }^{34}$ A. D. Becke, Phys. Rev. A 38, 3098 (1988).

${ }^{35}$ S. Grimme, J. Comp. Chem. 27, 1787 (2006).

${ }^{36}$ A. D. Boese, and N. C. Handy, J. Chem. Phys. 114, 5497 (2001).

${ }^{37}$ J. P. Perdew, K. Burke, and M. Ernzerhof, Phys. Rev. Lett. 77, 3865 (1996); ibid Phys. Rev. Lett. 78, 1396 (1997).

${ }^{38}$ J. P. Perdew, Phys. Rev. B 33, 8822 (1986).

${ }^{39}$ J. P. Perdew, J. A. Chevary, S. H. Vosko, K. A. Jackson, M. R. Pederson, D. J. Singh, and C. Fiolhais, Phys. Rev. B 46, 6671 (1992).

${ }^{40}$ R. Peverati, Y. Zhao, and D. G. Truhlar, J. Phys. Chem. Lett. 2, 1991 (2011).

${ }^{41}$ R. Peverati, and D. G. Truhlar, J. Chem. Theory Comput. 8, 2310 (2012).

${ }^{42}$ Y. Zhao, and D. G. Truhlar, J. Chem. Phys. 125, 194101 (2006).

${ }^{43}$ J. M. Tao, J. P. Perdew, V. N. Staroverov, and G. E. Scuseria, Phys. Rev. Lett. 91, 146401 (2003).

${ }^{44}$ A. D. Boese, and N. C. Handy, J. Chem. Phys. 116, 9559 (2002).

${ }^{45}$ T. van Voorhis, and G. E. Scuseria, J. Chem. Phys. 109, 400 (1998).

${ }^{46}$ A. D. Becke, J. Chem. Phys. 104, 1040 (1996).

${ }^{47}$ R. Peverati, and D. G. Truhlar, J. Phys. Chem. Lett. 3, 117 (2012).

${ }^{48}$ R. Peverati, and D. G. Truhlar, Phys. Chem. Chem. Phys. 10, 13171 (2012).

${ }^{49}$ A. D. Becke, J. Chem. Phys. 98, 1372 (1993).

${ }^{50}$ C. Adamo, and V. Barone, J. Chem. Phys. 110, 6158 (1999).

${ }^{51}$ F. A. Hamprecht, A. J. Cohen, D. J. Tozer, and N. C. Handy, J. Chem. Phys. 109, 6264 (1998).

${ }^{52}$ H. L. Schmider, and A. D. Becke, J. Chem. Phys. 108, 9624 (1998).

${ }^{53}$ X. Xu, Q. Zhang, R. P. Muller, and W. A. Goddard, J. Chem. Phys. 122, 014105 (2005).

${ }^{54}$ R. Peverati, and D. G. Truhlar, J. Chem. Phys. 135, 191102 (2011).

${ }^{55}$ Y. Zhao, N. E. Schultz, and D. G. Truhlar, J. Chem. Phys. 123, 161103 (2005).

${ }^{56}$ Y. Zhao, N. E. Schultz, and D. G. Truhlar, J. Chem. Theory Comput. 2, 364 (2006).

${ }^{57}$ V. N. Staroverov, G. E. Scuseria, J. Tao, and J. P. Perdew, J. Chem. Phys. 119, 12129 (2003).

${ }^{58}$ Y. Zhao, and D. G. Truhlar, J. Phys. Chem. A 109, 5656 (2005).

${ }^{59}$ S. Grimme, J. Chem. Phys. 124, 034108 (2006). 
${ }^{60}$ A. Tarnopolsky, A. Karton, R. Sertchook, D. Vuzman, and J. M. L. Martin, J. Phys. Chem. A 112, 3 (2008).

${ }^{61}$ S. Kozuch, D. Gruzman, and J. M. L. Martin, J. Phys. Chem. C 114, 20801 (2010).

${ }^{62}$ S. Kozuch, and J. M. L. Martin, Phys. Chem. Chem. Phys. 13, 20104 (2011).

${ }^{63}$ L. Goerigk, and S. Grimme, J. Chem. Theory Comput. 7, 291 (2011).

${ }^{64}$ T. Yanai, D. Tew, and N. Handy, Chem. Phys. Lett. 393, 51 (2004).

${ }^{65}$ O. A. Vydrov, and G. E. Scuseria, J. Chem. Phys. 125, 34109 (2006).

${ }^{66}$ J.-D. Chai, and M. Head-Gordon, J. Chem. Phys. 128, 084106 (2008).

${ }^{67}$ J.-D. Chai, and M. Head-Gordon, Phys. Chem. Chem. Phys. 10, 6615 (2008).

${ }^{68}$ R. Peverati, and D. G. Truhlar, J. Phys. Chem. Lett. 2, 2810 (2011).

${ }^{69}$ S. Grimme, S. Ehrlich, and L. Goerigk, J. Comput. Chem. 32, 1456 (2011).

${ }^{70}$ S. Grimme, J. Antony, S. Ehrlich, and H. Krieg, J. Chem. Phys. 132, 154104 (2010).

${ }^{71}$ S. Grimme, WIREs Comput. Mol. Sci. 1, 211 (2011).

72 A. D. Becke, and E. R. Johnson, J. Chem. Phys. 123, 154101 (2005).

73 ORCA is a modern electronic structure program package written by F. Neese, with contributions from Ute Becker, Dmytro Bykov, Dmitry Ganyushin, Andreas Hansen, Robert Izsak, Dimitrios G. Liakos, Christian Kollmar, Simone Kossmann, Dimitrios A. Pantazis, Taras Petrenko, et al. Max Planck Institute for Chemical Energy Conversion, 2014.

${ }^{74}$ F. Neese, WIREs Comput. Mol. Sci. 2, 73 (2012).

${ }^{75}$ L. A. Curtiss, P. C. Redfern, and K. Raghavachari, J. Chem. Phys. 126, 084108 (2007).

${ }^{76}$ L. A. Curtiss, P. C. Redfern, and K. Raghavachari, J. Chem. Phys. 127, 124105 (2007).

${ }^{77}$ B. Chan, J. Deng, and L. Radom, J. Chem. Theory Comput. 7, 112 (2011).

${ }^{78}$ J. A. Montgomery Jr, M. J. Frisch, J. W. Ochterski, and G. A. Petersson, J. Chem. Phys. 110, 2822 (1999); ibid 112, 6532 (2000).

${ }^{79}$ J. W. Ochterski, G. A. Petersson, and J. A. Montgomery Jr, J. Chem. Phys. 104, 2598 (1996).

${ }^{80}$ S. Grimme, J. Chem. Phys. 118, 9095 (2003).

${ }^{81}$ M. Pitonak, P. Neogrady, J. Cerny, S. Grimme, and P. Hobza, Chem. Phys. Chem. 10, 282 (2009).

${ }^{82}$ A. Karton, and L. Goerigk, J. Comp. Chem. 36, 622 (2015).

${ }^{83}$ S. Grimme, J. Comp. Chem. 24, 1529 (2003).

${ }^{84}$ T. Takatani, E. E. Hohenstein, and C. D. Sherrill, J. Chem. Phys. 128, 124111 (2008).

${ }^{85}$ M. Pitonak, J. Rezac, and P. Hobza, Phys. Chem. Chem. Phys. 12, 9611 (2011). 
${ }^{86}$ A. Karton, and J. M. L. Martin, J. Chem. Phys. 133, 144102 (2010).

${ }^{87}$ A. Karton, I. Kaminker, and J. M. L. Martin, J. Phys. Chem. A 113, 7610 (2009).

${ }^{88}$ M. L. Leininger, W. D. Allen, H. F. Schaefer, and C. D. Sherrill, J. Chem. Phys. 112, 9213 (2000).

${ }^{89}$ L.-J. Yu, and A. Karton, Chem. Phys. 441, 166 (2014).

${ }^{90}$ L.-J. Yu, F. Sarrami, A. Karton, and R. J. O’Reilly, Mol. Phys. 113, 1284 (2015).

${ }^{91}$ A. Karton, R. J. O’Reilly, B. Chan, and L. Radom, J. Chem. Theory Comput. 8, 3128 (2012).

${ }^{92}$ S. Grimme, L. Goerigk, and R. F. Fink, WIREs Comput. Mol. Sci. 2, 886 (2012).

${ }^{93}$ A. Halkier, T. Helgaker, P. Jørgensen, W. Klopper, H. Koch, J. Olsen, and A. K. Wilson, Chem. Phys. Lett. 286, 243 (1998).

${ }^{94}$ P. Jurecka, J. Sponer, J. Cerny, and P. Hobza, Phys. Chem. Chem. Phys. 8, 1985 (2006).

${ }^{95}$ D. G. Liakos, and F. Neese, J. Phys. Chem. A 116, 4801 (2012).

${ }^{96}$ B. Brauer, M. K. Kesharwani, and J. M. L. Martin, J. Chem. Theory Comput. 10, 3791 (2014).

${ }^{97}$ P. Jurečka, and P. Hobza, Chem. Phys. Lett. 365, 89 (2002).

${ }^{98}$ X. He, L. Fusti-Molnar, and K. M. Merz, Jr., J. Phys. Chem. A 113, 10096 (2009).

${ }^{99}$ R. J. O’Reilly, A. Karton, and L. Radom, Int. J. Quantum Chem. 116, 1862 (2012). 


\section{Graphical TOC}

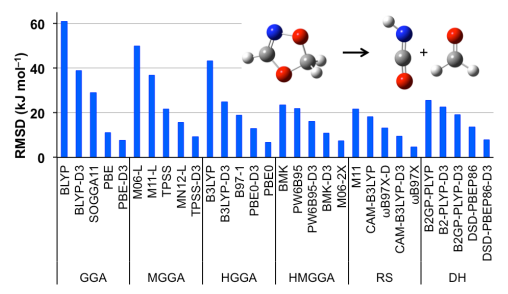

